\title{
Clinical and pathological disappearance of peritoneal dissemination in a patient with advanced gastric cancer receiving chemotherapy with S-1 and low-dose cisplatin
}

\author{
Yukako Akatsu ${ }^{1}$, Yoshiro Saikawa ${ }^{1}$, Tetsuro Kubota ${ }^{1}$, Masashi Yoshida $^{1}$, Toshiharu Furukawa ${ }^{1}$, \\ Yoshinide Otani ${ }^{1}$, Koichiro Kumai ${ }^{2}$, and Masaki Kitajima ${ }^{1}$ \\ ${ }^{1}$ Department of Surgery, Keio University School of Medicine, 35 Shinanomachi, Shinjuku-ku, Tokyo 160-8582, Japan \\ ${ }^{2}$ Center for Diagnostic and Therapeutic Endoscopy, Keio University School of Medicine, Tokyo, Japan
}

\begin{abstract}
A 54-year-old woman with severe abdominal distention suffered from massive ascites. Cytological examination revealed adenocarcinoma cells, leading to a diagnosis of peritonitis carcinomatosa. Gastrointestinal fiberscopy (GIF) resulted in a histological diagnosis of type 4 advanced gastric cancer with signet-ring cell carcinoma. The clinical diagnosis was confirmed to be cT3(SE)cN1cM0cH0cP1, cStage IV gastric cancer, type 4, according to the Japanese classification of gastric carcinoma. The patient was treated with S-1 and low-dose cisplatin (CDDP) in order to alleviate the critical state of the disease. After the third cycle of the regimen, the clinical response of $P 1$ was classified as a partial response (PR) according to the World Health Organization (WHO) criteria. The patient's appetite loss and abdominal discomfort were markedly alleviated. The patient experienced grade 2 leukocytopenia throughout the regimen. Surgery was performed. Ascites and peritoneal disseminated lesions were not observed, and cytological examination of the peritoneal washes was negative. Total gastrectomy with D1 lymph node dissection was performed, and the surgical diagnosis was sT3(SE)sN0sM0sH0sP0; sStage II. Microscopically, viable cancer cells were found to be scattered throughout the subserosal-serosal layers in the resected stomach. All of the samples from lesions that were potentially cancers involving peritoneal dissemination were diagnosed as fibrous scar tissues without any viable cancer cells. The patient is alive without recurrence at 10 months after surgery and 14 months after the initial chemotherapy. Thus, systemic chemotherapy with S-1/low-dose CDDP achieved desirable control of peritoneal disseminated cells, as assessed microscopically, suggesting that the regimen may be an effective strategy for the treatment of advanced gastric cancer with peritonitis carcinomatosa.
\end{abstract}

Key words Peritoneal dissemination - Gastric cancer - S-1 · Cisplatin

Offprint requests to: Y. Saikawa

Received: November 27, 2003 / Accepted: March 22, 2004

\section{Introduction}

Advanced gastric cancer, especially stage IV gastric cancer, has been recognized as a critical disease that is difficult to treat successfully by surgery. Five-year survival rates for surgical treatment alone are poor, and therefore gastric cancer often results in death $[1,2]$. On the other hand, several studies of novel anticancer agents, including S-1 (see below), taxoids, and irinotecan (CPT-11) have demonstrated that these agents have better efficacy against highly advanced gastric cancer than the conventional chemotherapeutic agents such as mitomycin $\mathrm{C}$, doxorubicin, or 5-fluorouracil (5-FU). However, a consistent standard chemotherapy has yet to be determined for cases of advanced gastric cancer [3]. The effectiveness of 5-FU alone in treating gastric cancer was reported to be only $10 \%-20 \%[3,4]$. Combination chemotherapy of 5-FU and cisplatin (CDDP) is one of the common regimens used in gastric cancer at present, and this combination shows a 30\%$40 \%$ efficacy rate against gastric cancer [5-7].

Recently, S-1 has been developed as a new oral agent derived from 5-FU. S-1 consists of tegafur, gimeracil, and oteracil. Gimeracil (5-chloro-2, 4dihydropyrimidine) is an agent that inhibits dihydropyrimidine dehydrogenase (DPD), which degrades 5-FU, while oteracil (monopotassium 1, 2, 3, 4tetrahydro-2, 4-dioxo-1, 3, 5-triazine-6 carboxylate) reduces the gastrointestinal toxicity induced by the phosphorylation of 5-FU [8-10]. Japanese late phase II trials of S-1 for the treatment of gastric cancer showed a high success rate for S-1 alone, with an overall response rate of up to $40 \%$. The incidence rate for adverse effects was around $20 \%$, and severe adverse effects were very rare [11-13]. This suggests that S-1 may be useful to improve the prognosis for patients with advanced gastric cancer, while allowing them to maintain a reasonably high quality of life. In addition, we previously demonstrated high efficacy, ranging to over $60 \%$, 
against stage IV gastric cancer for preoperative therapy consisting of a combination of S-1 plus low-dose CDDP [14]. The combination of 5-FU with CDDP has been recognized as a standard regimen in gastric cancer, because the antitumor activity of 5-FU is enhanced by the alteration of intracellular folate metabolism induced by CDDP. We also expected significant efficacy for the combination of S-1 with CDDP, and this therapy has been shown previously to have a high efficacy rate, with moderate toxicity [14-16].

We herewith report a stage IV gastric cancer patient with peritonitis carcinomatosa who was successfully treated with S-1 and low-dose CDDP chemotherapy preoperatively, followed by curative surgery. We observed complete histological disappearance of disseminated peritoneal lesions and malignant ascites. Thus, we have demonstrated the high potency of S-1 and low-dose CDDP chemotherapy against advanced gastric cancer, even when it is associated with peritonitis carcinomatosa.

\section{Case report}

\section{Drugs and definitions}

Combination chemotherapy of S-1 with low-dose CDDP was given to a stage IV gastric cancer patient with peritonitis carcinomatosa. Definitions of the Japanese Gastric Cancer Association [17] were utilized in staging and in both the radiological and the histological evaluations of treatment effects. The toxicity of S-1 and low-dose CDDP was classified using the common toxicity criteria CTC Cancer Therapy Evaluation Program, version 2.0 [18].

\section{Evaluation before therapy}

A 54-year-old woman with severe abdominal distention was evaluated. An abdominal computed tomography (CT) scan showed thickness of the gastric wall, massive ascites, pleural effusion, and infiltrating shadow around the omentum. Swollen lymph nodes were also observed around the stomach (Fig. 1). Cytological examination of the ascites revealed adenocarcinoma cells that were visualized microscopically, resulting in a diagnosis of peritonitis carcinomatosa. Gastrointestinal fiberscopy (GIF) showed fold thickness and gastric wall stiffness throughout the whole stomach that was compatible with a diagnosis of type 4 advanced gastric cancer. The cancer was diagnosed histologically as signet-ring cell carcinoma in biopsied specimens (Fig. 2). The final diagnosis was determined to be cStage IV gastric cancer, type 4 (cT3cN1cM0cH0cP1), with peritonitis carcinomatosa, according to the Japanese classification of gastric carcinoma. The serum levels of carcinoembryonic antigen

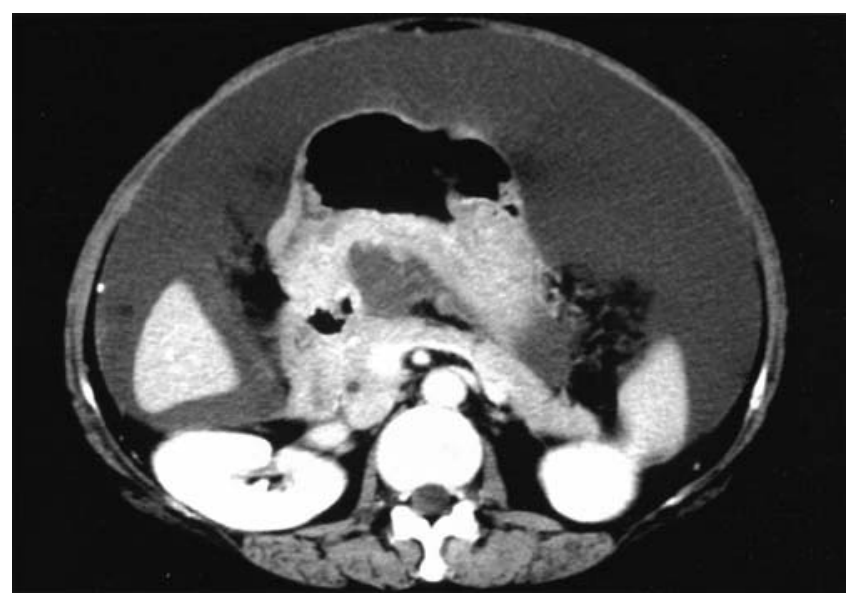

Fig. 1. Computed tomography (CT) findings before chemotherapy showed thickness of the gastric wall, massive ascites, and an infiltrating shadow around the omentum. Swollen lymph nodes were also observed around the stomach

(CEA) and carbohydrate antigen (CA)19-9 were nor$\mathrm{mal}$, at $1.3 \mathrm{U} / \mathrm{ml}$ and $1.0 \mathrm{U} / \mathrm{ml}$, respectively. The patient gave informed consent to be treated with a chemotherapeutic regimen in an attempt to alleviate the critical state of the disease.

\section{The first cycle of combination chemotherapy}

One cycle of the chemotherapy schedule consisted of 3 weeks of treatment. S-1 was administered orally at a dose of $100 \mathrm{mg} /$ day $\left(60 \mathrm{mg} / \mathrm{m}^{2}\right.$ per day) every day for 21 days. CDDP was infused at a dose of $10 \mathrm{mg} /$ day $(6 \mathrm{mg} /$ $\mathrm{m}^{2}$ per day) for $1 \mathrm{~h}$ on days $1-5,8-12$, and $15-19$, but CDDP was not infused on days $6,7,13,14,20$, or 21. While the total dose of CDDP was $90 \mathrm{mg} / \mathrm{m}^{2}$ when given in intermittent administrations over a 3-week period, daily administration of CDDP was given at a low dose $\left(6 \mathrm{mg} / \mathrm{m}^{2}\right.$ per day), resulting in low toxicity, as evaluated by renal dysfunction, nausea, or appetite loss. After one cycle of the regimen, GIF and an abdominal CT scan were performed to evaluate the clinical response to the chemotherapy. Endoscopic findings showed that there was no change (NC) in the primary lesion. An abdominal CT scan showed disappearance of ascites, decreased thickness of the gastric wall, and decreased infiltration around the omentum (Fig. 3), and this was defined as a partial response (PR). Only grade 2 leukocytopenia was observed as an adverse effect in the first cycle. At the time, the total doses of S- 1 and CDDP were $2100 \mathrm{mg}$ and $150 \mathrm{mg}$, respectively.

\section{Additional chemotherapy in the second and third cycles}

The response to the first cycle of the combination therapy was evaluated for effectiveness. The second and 


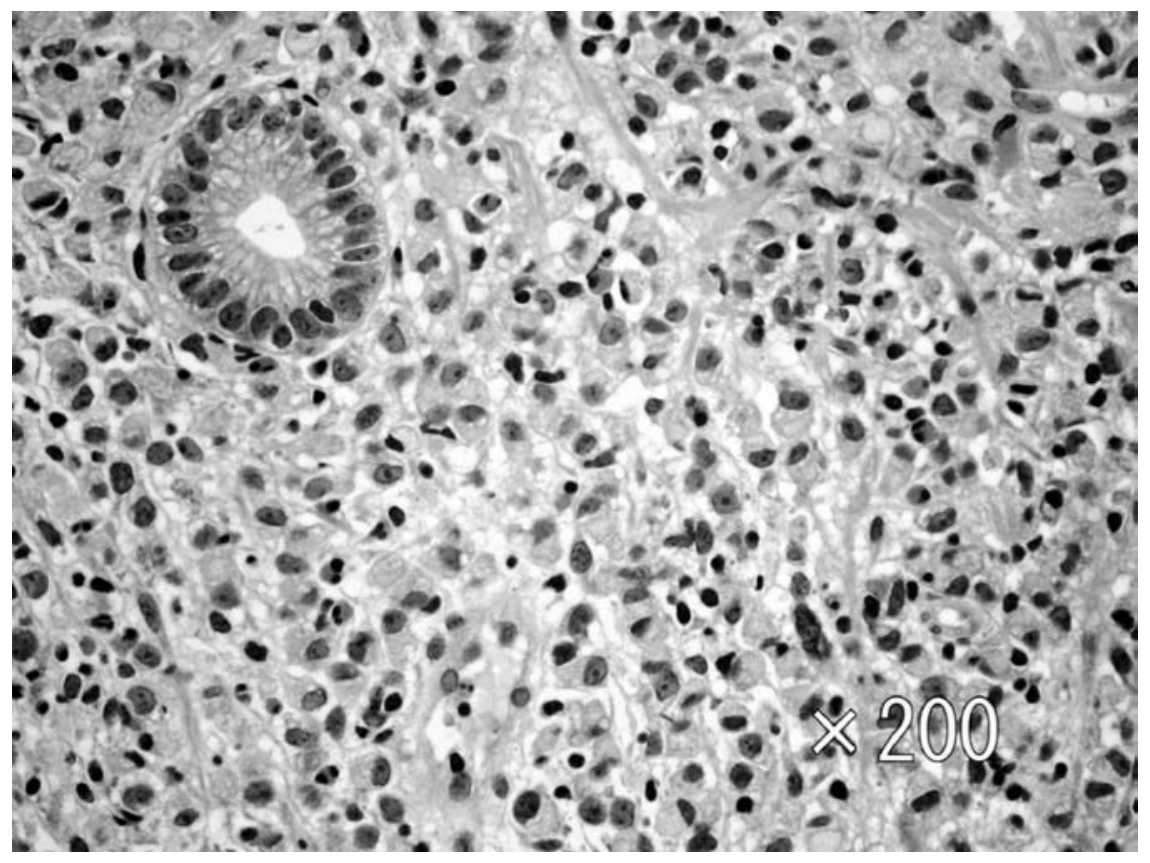

Fig. 2. Biopsy of a lesion before treatment showed signet-ring cell carcinoma. $\mathrm{H} \& \mathrm{E}$

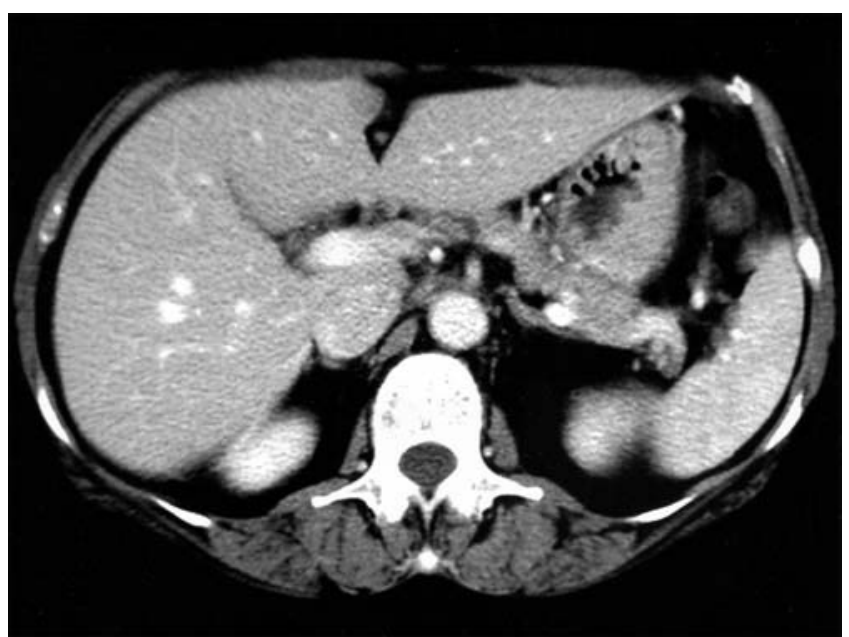

Fig. 3. CT after one cycle of the chemotherapy regimen showed disappearance of ascites, decreased thickness of the gastric wall, and decreased infiltration around the omentum

third cycles were then performed, with at least 2-week intervals without the chemotherapeutic agents scheduled between each cycle. The same drug doses were administered, using the same regimen as the first cycle. After the third cycle of the regimen, GIF and an abdominal CT scan were performed to evaluate the final clinical response to the therapy. GIF showed that no ulcerative or tumor lesions were found, and gastric fold thickness and gastric wall stiffness were reduced. The discolored gastric wall had changed to a white fibrous scar, evaluated as no change (NC), while an endoscopic biopsy did not detect any viable cancer cells. An abdominal CT scan showed disappearance of the ascites, and of the infiltrating shadow around the omentum, and decreasing thickness of the gastric wall. This clinical response was classified as a partial response (PR) according to the WHO criteria [19]. Only grade 2 leukocytopenia was observed as an adverse effect through the second and third cycles. The total doses of S-1 and CDDP over this time period were $6300 \mathrm{mg}$ and $451 \mathrm{mg}$, respectively.

\section{Surgical findings and procedures}

Laparotomy showed no ascites and no peritoneal disseminated lesions, and cytological examination of the peritoneal washes showed that they were cancer-free. The primary tumor could not be identified at the time of surgery, while the serosal surface of the gastric wall was discolored with white fibrosis, and slight stiffness. In addition, white scars were scattered around the perigastric area, and no viable cancer cells were detected histologically in the lesions. No swollen lymph nodes were observed in the abdominal cavity, indicating that there were no lymph node metastases. Total gastrectomy with D1 lymph node dissection was performed, resulting in a surgical diagnosis of sT3 (SE) sN0sM0sH0sP0, sStage II.

\section{Histopathology}

The macroscopic appearance of the primary lesion showed that it was a type 3 lesion, and other lesions did 
not demonstrate the typical appearance of type 4 cancer, such as gastric wall thickness or stiffness (Fig. 4). Impressively, white discolored surface of the serosa was seen throughout the stomach.

Microscopically, no viable cancer cells were detected from the mucosal layer to the muscular layer, while

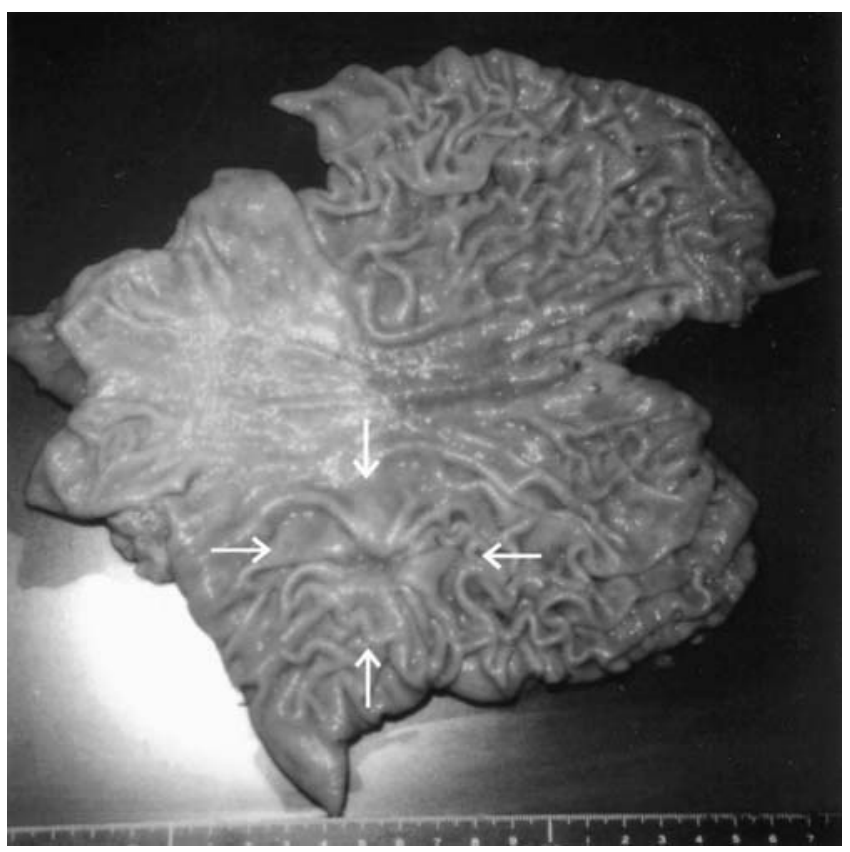

Fig. 4. Macroscopic findings of the resected specimen did not show any tumor lesions; furthermore, no typical characteristics of type 4 cancer (arrows), such as gastric wall thickness or stiffness, were observed throughout the whole stomach viable cancer cells were scattered throughout the subserosal-serosal layers in the resected stomach. Severe fibrosis was confirmed in all layers of the gastric wall and throughout the whole stomach (Fig. 5). Several lymph nodes showed fibrous changes without cancer cells, and no metastatic lymph nodes were detected histologically. All the samples from lesions that were suspected of being cancerous peritoneal disseminations were diagnosed as fibrous scar tissues, and were without any viable cancer cells.

\section{Postoperative course}

The postoperative course was uneventful with no surgical complications, and the patient was discharged 19 days after surgery. S-1 was utilized during outpatient postoperative adjuvant chemotherapy, and did not cause any side effects. The patient is alive without recurrence at 10 months after surgery and 14 months after the initial chemotherapy.

\section{Discussion}

As early gastrointestinal cancer is treated successfully by means of surgery alone, cancer chemotherapy is at present not applicable to early cancer at all. On the other hand, the clinical outcome of surgery alone is not acceptable in the case of advanced gastrointestinal cancer. Among gastric cancer patients, the 5-year survival rate for the group receiving surgery alone is less than $50 \%$ for stage III-IV patients, suggesting that new strat-

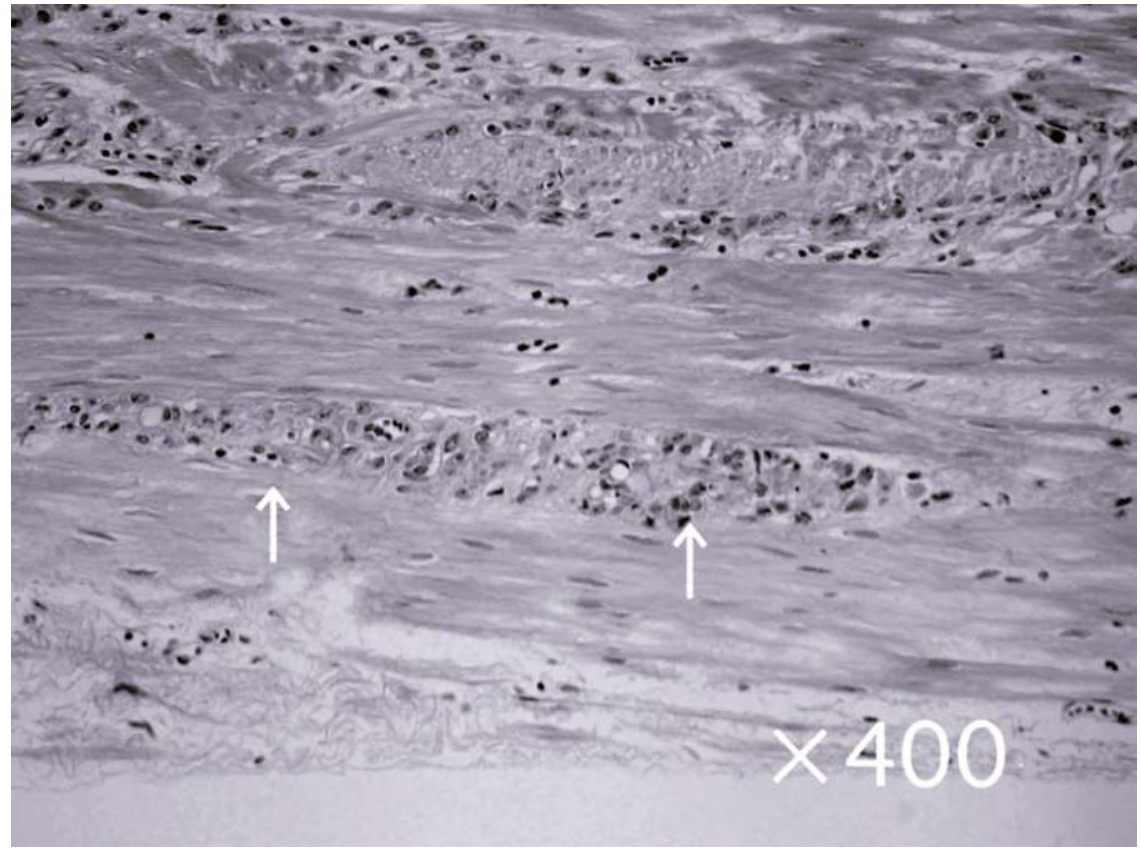

Fig. 5. Microscopic findings of the specimen showed that no visible cancer cells were detected from the mucosal layer to the muscular layer, while viable cancer cells (arrows) were scattered through the subserosal-serosal layers in the resected stomach. Severe fibrosis was confirmed in all layers of the gastric wall and throughout the whole stomach. This may have been caused by the replacement of the cancer lesions by fibrosis after severe inflammation and immunological reactions were evoked by chemotherapy against the cancer cells. $H \& E$ 
egies with or without surgery must be investigated to overcome this disease.

The recent development of novel cancer chemotherapeutic agents, such as taxoids, CPT-11, and S-1 has increased the potency of available therapies to control advanced stages of cancer. Moreover, several reports have shown the clinical effectiveness of these agents applied alone or as part of a combination therapy against advanced gastrointestinal cancer. A phase I-II study of S-1 has already been performed for patients with advanced gastric cancer in Japan, revealing a good clinical response. S-1 alone had up to $50 \%$ efficacy, and there were few severe adverse reactions [12]. S-1, when given alone, showed a high response rate, of approximately $40 \%-50 \%$ [11,12], and had low toxicity in several clinical studies of gastric cancer. The clinical effects of 5-FU are known to be enhanced by combination with CDDP in several kinds of carcinoma. Previously, in a pilot study, we attempted to evaluate a new preoperative combination chemotherapy against highly advanced gastric carcinoma, using S-1 and low-dose CDDP, and this resulted in a good response preoperatively in patients with stage IV gastric cancer [14]. In that study, six of ten patients who underwent surgical resection following the chemotherapy showed a pathological response above grade $1 \mathrm{~b}$ in either the stomach or lymph nodes. Further studies, including randomized controlled studies, will be necessary to evaluate the efficacy of the preoperative chemotherapy. Since 2000, we have demonstrated the significant effectiveness of this combination, and we have also reported that a high histological response against advanced gastric cancer was effected by the S-1/low-dose CDDP regimen [16], the same as that given to the patient in this report.

$\mathrm{S}-1$ is a $5-\mathrm{FU}$ derivative, and we designed the regimen to include S-1 and low-dose CDDP, because it is well known that the antitumor effects of 5-FU are modulated by CDDP. Furthermore, the clinical effectiveness of the 5-FU and CDDP combination has already been recognized, and this combination is one of the standard therapies for gastric cancer [5-7]. One mechanism for the activity of this combination is as follows. CDDP inhibits the incorporation of exogenous L-methionine into cancer cells and increases the level of reduced folates, which are essential cofactors for the formation of a tight ternary complex of thymidylate synthase and 5-fluoro-2'-deoxyuridine-5'-monophosphatase. This results in the enhancement of the anticancer activity of 5FU [6]. In the S-1/CDDP regimen, the main anticancer agent is S-1, while low-dose CDDP may be a biochemical modulator of S-1. Gimeracil in S-1 is known to be a potent inhibitor of 5-FU degradation, inhibiting DPD about 200 times more effectively than uracil. This leads to the prolonged presence of 5-FU in the serum $[11,15]$, and results in high efficacy against cancer. Oteracil in S1 reduces the gastrointestinal toxicity induced by the phosphorylation of 5-FU [15]. Thus, the incidence of adverse reactions with pathological grade 3 and 4 cancers was less than $20 \%$, and severe adverse reactions were not observed when S-1 alone was administered [15].

The patient described in the present report experienced only grade 2 leukocytopenia throughout the period of administration of the regimen. This was due to the use of granulocyte-colony stimulating factor. Concomitantly, she experienced decreased abdominal distention, increased appetite, and improved quality of life in response to this regimen. It is unclear whether or not surgical resection should be performed for such a highly advanced gastric cancer with peritoneal carcinomatosa, considering the patient's prognosis and quality of life. Although this patient chose surgery, the clinical outcome of the surgery was still uncertain, while alleviation of the critical state of the disease by the chemotherapy was confirmed clinically. Surgery was completed successfully without any postoperative complications, and there was no undesirable event after surgery, and therefore no prolonged hospitalization. Thus, the preoperative administration of S-1 and CDDP appears to be relatively well tolerated, even before the surgery is performed.

Some remaining cancer cells were observed, and these were scattered particularly in the serosal layer, while massive tumor formations with viable cancer cells were not detected in the patient's stomach. Severe fibrosis was confirmed in all layers of the gastric wall and throughout the whole stomach. This may have been caused by the replacement of the cancer lesions by fibrosis after severe inflammation and immunological reactions were evoked by the chemotherapy against the cancer cells. We are not sure whether the decreased number of cancer cells in response to the chemotherapy means that the chemotherapy improved the prognosis of the type 4 scirrhous gastric cancer (which is reported to show high malignant potential, being associated with peritoneal dissemination and lymph node metastasis). Accordingly, the patient's prognosis is still uncertain at present, although histological evaluation of specimens from the white-scarred peritoneum that were suspected to be disseminated peritoneal cancers revealed no viable cancer cells. However, at the very least, the patient has obtained 14 months of survival after the initial treatment by means of systemic chemotherapy with S-1/low-dose CDDP, and she has maintained a good quality of life without disease recurrence. This encourages us to suggest that this regimen may be an effective strategy for the treatment of highly advanced gastric cancer with peritonitis carcinomatosa. 


\section{References}

1. Hundahl SA, Phillips JL, Menck HR. The National Cancer Data Base report on poor survival of US gastric carcinoma patients treated with gastrectomy: fifth edition. American Joint Committee on Cancer Staging, Proximal Disease, and the "different disease" hypothesis. Cancer 2000;88:921-32.

2. Comis RI, Carter SK. Integration of chemotherapy into combined modality treatment of solid tumors. III. Gastric cancer. Cancer Treat Rev 1974;1:221-38.

3. Moynihan T, Hansen R, Anderson T. Continuous 5-fluorouracil infusion in advanced gastric carcinoma. Am J Clin Oncol 1988; 11:461-4

4. Lokich JJ, Ahlgren JD, Gullo JJ, Philips JA, Fryer JG. A prospective randomized comparison of continuous infusion fluorouracil with a conventional bolus schedule in metastatic colorectal carcinoma: a Mid-Atlantic Oncology Program Study. J Clin Oncol 1989;7:425-32.

5. Chung YS, Yamashita Y, Inoue T, Matsuoka T, Nakata B, Onoda $\mathrm{N}$, et al. Continuous infusion of 5-fluorouracil and low dose cisplatin infusion for the treatment of advanced and recurrent gastric adenocarcinoma. Cancer 1997;80:1-7.

6. Shirasaka T, Shimamoto Y, Ohshiro H, Saito H, Fukushima M. Metabolic basis of the synergistic antitumor activities of 5fluorouracil and cisplatin in rodent tumor models in vivo. Cancer Chemother Pharmacol 1993;32:167-72.

7. Kondo K, Murase M, Kodera Y, Akiyama S, Ito K, Yokoyama Y, et al. Feasibility study on protracted infusion 5-fluorouracil and consecutive low-dose cisplatin for advanced gastric cancer. Oncology 1996;53:64-7.

8. Harris BE, Song R, Soong ST, Diasio RB. Relationship between dihydropyrimidine dehydrogenase activity and plasma drug levels in cancer patients receiving 5-fluorouracil by protected continuous infusion. Cancer Res 1990;50:197-201.

9. Shirasaka T, Shimamoto Y, Ohshimo H, Yamaguchi M, Kato T, Yonekura K, et al. Development of a novel form of an oral 5fluorouracil derivative (S-1) directed to the potentiation of the tumor selective cytotoxicity of 5-fluorouracil by two biochemical modulators. Anticancer Drugs 1996;7:548-57.

10. Shirasaka K, Shimamoto Y, Fukushima M. Inhibition by oxanic acid of gastrointestinal toxicity of 5-fluorouracil without loss of its antitumor activity in rats. Cancer Res 1993;53:4004-9.

11. Sakata Y, Ohtsu A, Horikoshi N, Sugimachi K, Mitachi Y, Taguchi T. Late phase II study of novel oral fluoropyrimidine anticancer drug S-1 (1 M tegafur- $0.4 \mathrm{M}$ gimestat- $1 \mathrm{M}$ otastat potassium) in advanced gastric cancer patients. Eur J Cancer 1998;34:1715-20.

12. Sugimachi K, Maehara Y. The S-1 Gastrointestinal Cancer Study Group. An early phase II study of oral S-1, a newly developed 5fluorouracil derivative for advanced and recurrent gastrointestinal cancers. Oncology 1999;57:202-10.

13. Koizumi W, Kurihara M, Nakano S, Hasegawa K. The S-1 Gastrointestinal Cancer Study Group: phase II study of S-1, a novel derivative of 5-fluorouracil, in advanced gastric cancer. Oncology 2000;58:191-7.

14. Saikawa Y, Akasaka Y, Kanai T. Preoperative combination chemotherapy with S-1 and low dose cisplatin against highly advanced gastric cancer. Oncol Rep 2002;10:381-6.

15. Iwahashi M, Nakamori M, Tani M, Yamaue H, Sakaguchi S, Nakamura M, et al. Complete response of highly advanced gastric cancer with peritoneal dissemination after new combined chemotherapy of S-1 and low-dose cisplatin: report of a case. Oncology 2001;61:16-22.

16. Yoshimizu N, Saikawa Y, Kubota T, Akiba Y, Yoshida M, Otani $\mathrm{Y}$, et al. A case report: complete response of a highly advanced gastric carcinoma to preoperative chemo-radiotherapy with S-1 and low dose cisplatin. Gastric Cancer 2003;3:191-6.

17. Japanese Gastric Cancer Association. Introduction to JGCA gastric cancer treatment guidelines. Tokyo: Kanehara; 2001. p. 46.

18. Official reference of CTC version 2.0. Cancer Therapy Evaluation Program. Common Toxicity Criteria, Version 2.0. DCTD, NCI, NIH, DHHS 1998.

19. Miller AB, Hoogstraten B, Staquet M, Winkler A. Reporting results of cancer treatment. Cancer 1981;47:207-14. 